\title{
Unexpected complexity of multidrug resistance in the mcr-1-harbouring Escherichia coli
}

\author{
Rongsui $\mathrm{Gao}^{1 \dagger}$, Yue $\mathrm{Li}^{2 \dagger}$, Jingxia $\operatorname{Lin}^{1}$, Chen $\operatorname{Tan}^{2 *} \&$ Youjun Feng ${ }^{1 \dagger}$ \\ ${ }^{1}$ Department of Medical Microbiology and Parasitology, Zhejiang University School of Medicine, Hangzhou 310058, China; \\ ${ }^{2}$ State Key Laboratory of Agricultural Microbiology, College of Veterinary Medicine, Huazhong Agricultural University, Wuhan 430070, \\ China
}

Received April 17, 2016; accepted May 6, 2016; published online May 12, 2016

Citation: Gao, R., Li, Y., Lin, J., Tan, C., and Feng, Y. (2016). Unexpected complexity of multidrug resistance in the $m c r-1$-harbouring Escherichia coli. Sci China Life Sci 59, 732-734. doi: 10.1007/s11427-016-5070-1

Dear Editor,

The discovery that the mobile colistin resistance gene ( $m c r-1)$ is encoded by plasmids and is prevalent in food animals and human beings worldwide (Hasman et al., 2015; Liu et al., 2015) has challenged greatly our traditional idea that polymyxin (consisting of two isoforms: polymyxin B and polymyxin E (colistin)) acts as an ultimate line of refuge in the clinical treatment against the severe infections by the multidrug-resistant Gram-negative pathogens (Nation et al., 2015; Paterson and Harris, 2015). To make matters worse, the $\mathrm{mcr}-1$ gene was recently found to be co-localized with other drug resistance genes in the plasmid pKH457-3-BE with an IncP backbone from a bovine isolate in Belgium (Surbi Malhotra-Kumar, 2016), which is far different from the pig-isolated plasmid, pHNSHP45 with an IncI2 backbone (Liu et al., 2015). It raised the possibility that super-bugs with pan-drug resistance might be emerging.

Given the fact that (1) genomic sequences of the mcr-1-harbouring plasmids are extremely limited right now, and (2) co-occurrence of MCR-1-mediated colistin resistance with other multidrug resistance remains unclear, we screened a collection of antibiotic-resistant isolates (no., 102) from swine tissues in China. In particular, only 6 of the

$\dagger$ Contributed equally to this work

*Corresponding author (email: tanchen@mail.hzau.edu.cn)

†Corresponding author (email: fengyj@zju.edu.cn)
16 colistin-resistant isolates (namely WH01, WH02, ..., WH16) are verified to be mcr-1-positive Escherichia coli (Figure 1A, 1B and S1). They are namely WH03, WH07, WH09, WH12, WH13, and WH15. In contrast, the majority of the isolates we checked is $m c r$-l-negative, but remains appreciable level of the colistin resistance (not shown), implying the possibility that some mystical machineries/vectors claim for this antibiotic resistance. Subsequently, we tested the sensitivity of the six $m c r-1$-positive $E$. coli isolates to a dozen of various antibiotics (Figure $1 \mathrm{C}$ and $\mathrm{S} 1)$. The antibiotics (15 in total) we used here are categorized into eight groups 1: $\beta$-lactams antibiotics including ampicillin (AMP), cefotaxime (CTX); 2: quinolone antibiotic such as ciprofloxacin (CIP), norfloxacin (NOR) and levofloxacin (LEV); 3: tetracycline antibiotics (tetracycline (TET) and doxycycline (DOX)); 4: aminoglycoside antibiotics like amikacin (AMK), gentamycin (GEN) and kanamycin (KAN); 5: amino alcohol antibiotic, chloramphenicol (CHL); 6: sulfonamide antibiotic, trimethoprim (TMP); 7 : nitrofuran antibiotic, macrodantin (NFT); and 8: cationic polypeptide antibiotic, colistin (COL)).

To our surprise, the unexpected complexity of the multidrug resistance was observed in the $m c r$ - 1 -harbouring isolates. First, the $m c r$ - 1 -poitive isolate WH13 exhibited the mostly-broad-spectrum antibiotic resistance in that it can be tolerant with nearly all the 15 antibiotics with an exception of amikacin (Figure 1C). Second to the WH13, the mcr-1-carrying isolate $\mathrm{WH} 12$ are resistant to 13 antibiotics (such as cefotaxime, tetracycline and kanamycin) (Figure 

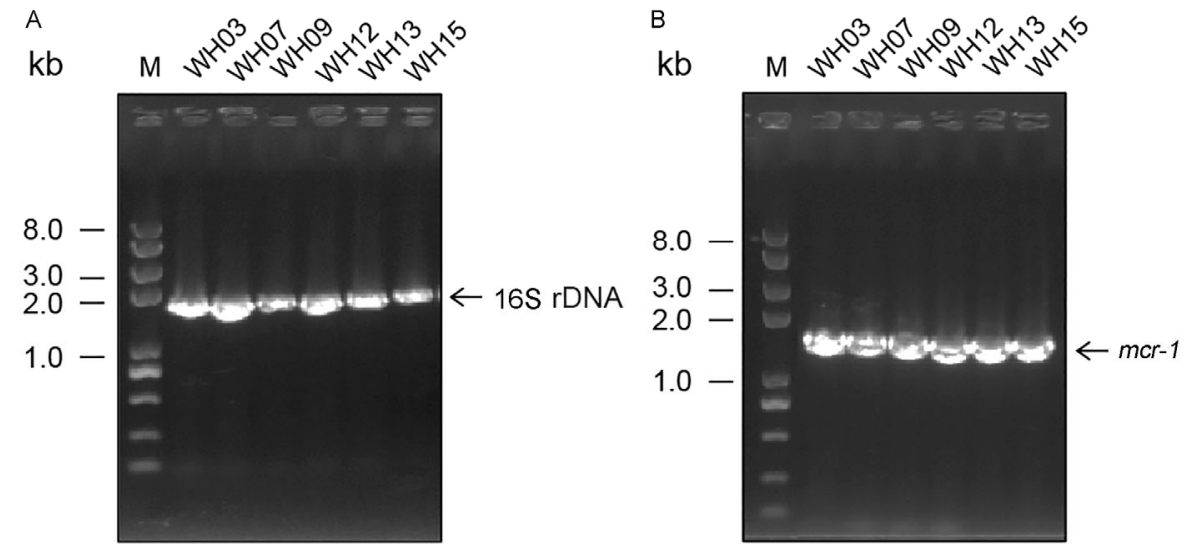

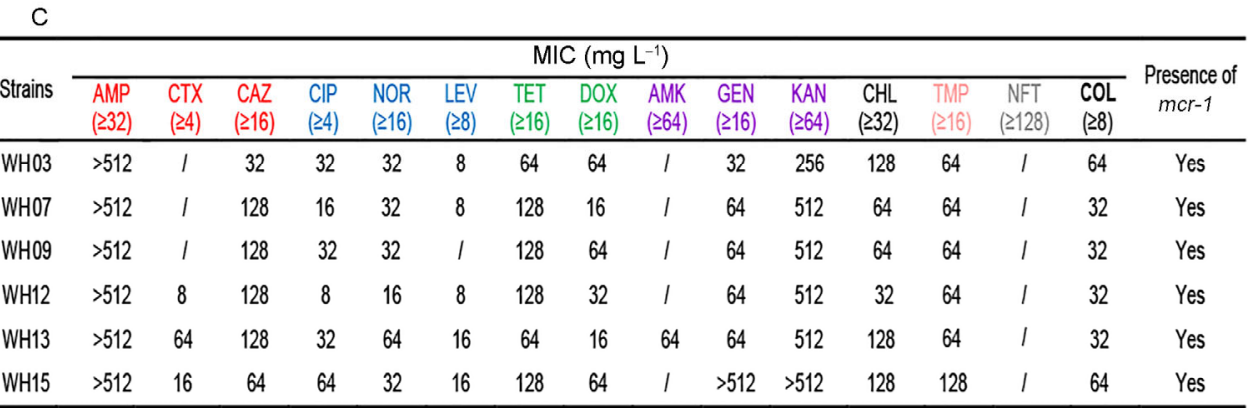

Figure 1 Complicated profile for the multi-drug resistance in mcr-1-positive Escherichia coli isolates from the swine lung microbiota. A, 16S-based PCR identification for Escherichia coli isolates from swine lung microbiota. B, PCR screening of colistin-resistant $E$. coli strains for the presence of the $m c r-1$ gene. The colistin-resistant bacterial strains are namely WH03, WH07, WH09, WH12, WH13, and WH15. The expected size of 16S rDNA is around $1.5 \mathrm{~kb}$, and the PCR amplicon of the $m c r-1$ gene in full length is about $1.6 \mathrm{~kb}$. kb, kilo-base pair; M, DNA marker. C, Unexpected diversity in multi-drug resistance profile of the $m c r-1$-harbouring swine $E$. coli isolates. $\beta$-lactams antibiotics are in red; quinolone antibiotic is in blue; tetracycline antibiotics are in green; aminoglycoside antibiotics are in purple; amino alcohol antibiotic is in black; Sulfonamide antibiotic is in pink; nitrofuran antibiotic is in grey; and polypeptide-like antibiotic is highlighted in bold letters. MIC, minimum inhibitory concentration; AMP, ampicillin; CTX, cefotaxime; CAZ, ceftazidime; GEN, gentamycin; KAN, kanamycin; AMK, amikacin; TET, tetracycline; DOX, doxycycline; CIP, ciprofloxacin; NOR, norfloxacin; LEV, levofloxacin; CHL, chloramphenicol; TMP, trimethoprim; NFT, macrodantin; and COL, colistin (polymyxin E). /, the value less than the cut-off.

1C). Third, the antibiotic resistance profile of the two isolates (WH03 and WH15) reached 9 and 12 antibiotics (Figure 1C). Finally, both isolates WH07 and WH09 showed the resistance to 7 antibiotics (Figure 1C). Of particular note, all the above colistin-resistant isolates still remain susceptible to the aminoglycoside antibiotics amikacin (Figure 1C). Apart from the strain WH13, the remaining five isolates (6 in total) can be effectively inhibited in the presence of the nitrofuran antibiotic, macrodantin (Figure 1C).

Our data provided a glimpse of complicated situation for multidrug resistance in the $m c r-1$-harbouring isolates with the origin of swine lung, suggesting the possibility that diversified $m c r-1$-carring plasmid reservoirs are present in swine lung microbiota. In fact, this speculation has already been verified in the $m c r$ - 1 -harbouring human isolates (Ye et al., 2016). Increasingly-accumulated evidence suggested that the $m c r-1$ gene might co-exist with the variants (NDM-5 and NDM-9) of NDM-1 (New Delhi-Metallo- $\beta$ lactamase 1), another notorious antidrug gene (Du et al., 2016; Yao et al., 2016). In addition, although it seemed likely that bacterial infections by these $m c r$ - 1 -harbouring pathogens with the multidrug resistance still can be con- trolled using correct antibiotic-based therapy, it is greatly needed to integrate combined strategies to minimize/control current situation that the multi-drug resistance is increasing worldwide including China.

Compliance and ethics The author(s) declare that they have no conflict of interest.

Acknowledgements This work was supported by Zhejiang Provincial Natural Science Foundation for Distinguished Young Scholars (LR15H190001), the National Natural Science Foundation of China (31570027), the start-up package from Zhejiang University (Youjun Feng), and the "Young 1000 Talents" Award (Youjun Feng).

Du, H., Chen, L., Tang, Y.W., and Kreiswirth, B.N. (2016). Emergence of the $m c r-1$ colistin resistance gene in carbapenem-resistant Enterobacteriaceae. Lancet Infect Dis 16, 287-288.

Hasman, H., Hammerum, A.M., Hansen, F., Hendriksen, R.S., Olesen, B., Agerso, Y., Zankari, E., Leekitcharoenphon, P., Stegger, M., Kaas, R.S., Cavaco, L.M., Hansen, D.S., Aarestrup, F.M., and Skov, R.L. (2015). Detection of mcr-1 encoding plasmid-mediated colistin-resistant Escherichia coli isolates from human bloodstream infection and imported chicken meat, Denmark 2015. Euro Surveill 20, ES.2015.20.49.30085. 
Liu, Y.Y., Wang, Y., Walsh, T.R., Yi, L.X., Zhang, R., Spencer, J., Doi, Y., Tian, G., Dong, B., Huang, X., Yu, L.F., Gu, D., Ren, H., Chen, X., Lv, L., He, D., Zhou, H., Liang, Z., Liu, J.H., and Shen, J. (2016). Emergence of plasmid-mediated colistin resistance mechanism MCR-1 in animals and human beings in China: a microbiological and molecular biological study. Lancet Infect Dis 16, 161-168.

Nation, R.L., Li, J., Cars, O., Couet, W., Dudley, M.N., Kaye, K.S., Mouton, J.W., Paterson, D.L., Tam, V.H., Theuretzbacher, U., Tsuji, B.T., and Turnidge, J.D. (2015). Framework for optimisation of the clinical use of colistin and polymyxin B: the Prato polymyxin consensus. Lancet Infect Dis 15, 225-234.

Paterson, D.L., and Harris, P.N. (2016). Colistin resistance: a major breach in our last line of defence. Lancet Infect Dis 16, 132-133.

Malhotra-Kumar, S., Xavier, B.B., Das, A.J., Lammens, C., Butaye, P., and Goossens, H. (2016). Colistin resistance gene $m c r-1$ harboured on a multidrug resistant plasmid. Lancet Infect Dis 16, 283-284.

Yao, X., Doi, Y., Zeng, L., Lv, L., and Liu, J.H. (2016). Carbapenemresistant and colistin-resistant Escherichia coli co-producing NDM-9 and MCR-1. Lancet Infect Dis 16, 288-289.

Ye, H., Li, Y., Li, Z., Gao, R., Zhang, H., Wen, R., Gao, G.F., Hu, Q., and Feng, Y. (2016). Diversified $m c r$-1-harbouring plasmid reservoirs confer resistance to colistin in human gut microbiota. MBio 7, pii: e00177-16. doi: 10.1128/mBio.00177-16

Open Access This article is distributed under the terms of the Creative Commons Attribution License which permits any use, distribution, and reproduction in any medium, provided the original author(s) and source are credited.

\section{SUPPORTING INFORMATION}

Figure S1 Gram-staining analyses for determination of mcr-1-positive E. coli isolates from the swine microbiota.

The supporting information is available online at life.scichina.com and link.springer.com. The supporting materials are published as submitted, without typesetting or editing. The responsibility for scientific accuracy and content remains entirely with the authors. 